Portland State University

PDXScholar

$9-1-2001$

\title{
Thermal Activation between Landau Levels in the Organic Superconductor $\boldsymbol{\beta}$ "-(BEDT- TTF)2SF5CH2CF2SO3
}

Gary L. Gard

Portland State University

M. S. Nam

A. Ardavan

J. A. Symington

J. Singleton

See next page for additional authors

Follow this and additional works at: https://pdxscholar.library.pdx.edu/chem_fac

Part of the Physics Commons

Let us know how access to this document benefits you.

\section{Citation Details}

Gard, Gary, Nam, M. -S., Ardavan, A., Symington, J. A., Singleton, N., Harrison, C. H., Mielke, J. A., Schlueter, J. A., and Winter, R. W. (2001) Thermal Activation between Landau Levels in the Organic Superconductor $\beta "$ "-(BEDT-TTF) ${ }_{2} \mathrm{SF}_{5} \mathrm{CH}_{2} \mathrm{CF}_{2} \mathrm{SO}_{3}$. Physical Review Letters, 87, 11700-1.

This Article is brought to you for free and open access. It has been accepted for inclusion in Chemistry Faculty Publications and Presentations by an authorized administrator of PDXScholar. Please contact us if we can make this document more accessible: pdxscholar@pdx.edu. 


\section{Authors}

Gary L. Gard, M. S. Nam, A. Ardavan, J. A. Symington, J. Singleton, N. Harrison, C. H. Mielke, J. A. Schlueter, and Rolf Walter Winter 


\title{
Thermal Activation between Landau Levels in the Organic Superconductor $\beta^{\prime \prime}$-(BEDT-TTF $)_{2} \mathrm{SF}_{5} \mathrm{CH}_{2} \mathrm{CF}_{2} \mathrm{SO}_{3}$
}

\author{
M.-S. Nam, ${ }^{1}$ A. Ardavan, ${ }^{1}$ J. A. Symington, ${ }^{1}$ J. Singleton,,${ }^{1,2}$ N. Harrison, ${ }^{2}$ \\ C. H. Mielke, ${ }^{2}$ J. A. Schlueter, ${ }^{3}$ R. W. Winter, ${ }^{4}$ and G. L. Gard ${ }^{4}$ \\ ${ }^{1}$ Department of Physics, University of Oxford, Clarendon Laboratory, Parks Road, Oxford OX1 3PU, United Kingdom \\ ${ }^{2}$ National High Magnetic Field Laboratory, LANL, MS-E536, Los Alamos, New Mexico 87545 \\ ${ }^{3}$ Chemistry and Materials Science Division, Argonne National Laboratory, Argonne, Illinois 60439 \\ ${ }^{4}$ Department of Chemistry, Portland State University, Portland, Oregon 97207
}

(Received 19 December 2000; published 23 August 2001)

\begin{abstract}
We show that Shubnikov-de Haas oscillations in the interlayer resistivity of the organic superconductor $\beta^{\prime \prime}$-(BEDT-TTF $)_{2} \mathrm{SF}_{5} \mathrm{CH}_{2} \mathrm{CF}_{2} \mathrm{SO}_{3}$ become very pronounced in magnetic fields $\sim 60 \mathrm{~T}$. The conductivity minima exhibit thermally activated behavior that can be explained simply by the presence of a Landau gap, with the quasi-one-dimensional Fermi surface sheets contributing negligibly to the conductivity. This observation, together with complete suppression of chemical potential oscillations, is consistent with an incommensurate nesting instability of the quasi-one-dimensional sheets.
\end{abstract}

DOI: 10.1103/PhysRevLett.87.117001

The quantizing effect of a magnetic field on a chargecarrier system is well known [1]. In metals, this leads to oscillations of the free energy and quasiparticle density of states as Landau levels cross the Fermi energy [2]. The effect is very pronounced in quasi-two-dimensional (Q2D) metals containing Fermi surfaces (FSs) that are approximately cylindrical [3]. Recently there has also been interest in analogous effects in quasi-one-dimensional (Q1D) FS sections, which can lead to magnetic-fieldinduced quantization [4] and localization [5].

In this paper we describe the magnetoresistance of the organic superconductor $\beta^{\prime \prime}$-(BEDT-TTF) ${ }_{2} \mathrm{SF}_{5} \mathrm{CH}_{2} \mathrm{CF}_{2} \mathrm{SO}_{3}$ $\left(T_{\mathrm{c}} \approx 5.4 \mathrm{~K}[6]\right)$. Band structure calculations suggest that this material possesses a FS comprising a Q2D cylinder and a pair of Q1D sheets [6]. However, Shubnikov-de Haas $(\mathrm{SdH})$ and de Haas-van Alphen (dHvA) measurements reveal that the Q2D cylinder has only one-third the expected cross section [7-9]. Angle-dependent magnetoresistance oscillation (AMRO) $[8,10]$ and millimeter-wave magnetoconductivity experiments [11] show that the cross section of this Q2D pocket resembles an elongated diamond. The same experimental techniques find no evidence for the presence of Q1D Fermi sheets at low temperatures $[8,10,11]$, unlike the situation in other Q2D organic metals [3]. By contrast, in order to explain the observation of a fixed chemical potential $\mu$ in the dHvA effect [7], Wosnitza et al. proposed Q1D states which have an enormous density of states, exceeding the estimates from band structure calculations by at least an order of magnitude [7]. Moreover, using a simple formula for the background magnetoresistance, Wosnitza et al. suggested that the Q1D sheets become localized in a magnetic field [12]. In the present paper, we show that magnetoresistance data suggest a much simpler explanation. The thermally activated behavior of the data at integer Landau level filling factors is explained entirely in terms of a Landau gap. Moreover, the failure of the Q1D sheets to contribute to the conductiv-
PACS numbers: 74.70.Kn, 71.20.Rv, 78.20.Ls

ity together with their ability to fix $\mu$ is explained by their nesting to form an incommensurate density-wave ground state. This mechanism is supported by the temperature dependence of the resistivity at $B=0$.

Single crystals $\left(\sim 1.0 \times 0.5 \times 0.2 \mathrm{~mm}^{3}\right)$ of $\beta^{\prime \prime}$-(BEDT$\mathrm{TTF})_{2} \mathrm{SF}_{5} \mathrm{CH}_{2} \mathrm{CF}_{2} \mathrm{SO}_{3}$ were prepared using standard electrochemical techniques [6]. Contacts were applied using $12.5 \mu \mathrm{m} \mathrm{Pt}$ wires and graphite paint, in a configuration which gives the interplane magnetoresistance, $\rho_{z z}$ [3]. Magnetic fields $B$ were provided by $60 \mathrm{~T}$ pulsed magnets at the National High Magnetic Field Laboratory (NHMFL), Los Alamos; two magnet pulses were required for each data set, the dc sample current of $5 \mu \mathrm{A}$ being reversed between pulses to remove contributions from thermoelectric and inductive voltages. Temperatures $T$ between 0.5 and $4.2 \mathrm{~K}$ were obtained using a ${ }^{3} \mathrm{He}$ cryostat. Sample heating was not found to be a problem. This was checked by ensuring that the measured $\rho_{z z}$ was independent of $d B / d t$ and by comparing data with those taken in quasistatic fields of up to $\sim 33 \mathrm{~T}$ at NHMFL Tallahassee.

Figure 1 shows the $T$-dependent resistivity $\rho_{z z} ; B$ was applied perpendicular to the Q2D planes. Pronounced $\mathrm{SdH}$ oscillations are visible, with a frequency $F=196 \pm 3 \mathrm{~T}$ in agreement with earlier data [7-9,11]. At high fields, $\rho_{z z}$ becomes very large close to integer Landau-level filling factor, $\nu=F / B$.

In three-dimensional (3D) metals exhibiting the $\mathrm{SdH}$ effect, $\Delta \sigma / \sigma$, the ratio of the oscillatory part of the conductivity to the background conductivity (originating from the rest of the FS) is proportional to the density of states, which is in turn proportional to $B^{2} d M / d B$, where $M$ is the magnetization [2]. This is valid in $3 \mathrm{D}$ metals because $\Delta \sigma$ is only a very weak perturbation of $\sigma$. However, this simple proportionality is no longer valid in a Q2D system in which $\Delta \sigma \gg \sigma$ [13], as is clearly the case in Fig. 1. For such data, the value of $\sigma$ that one extracts by comparing $\Delta \sigma / \sigma$ with $B^{2} d M / d B$ (as done in Ref. [12]) has no 


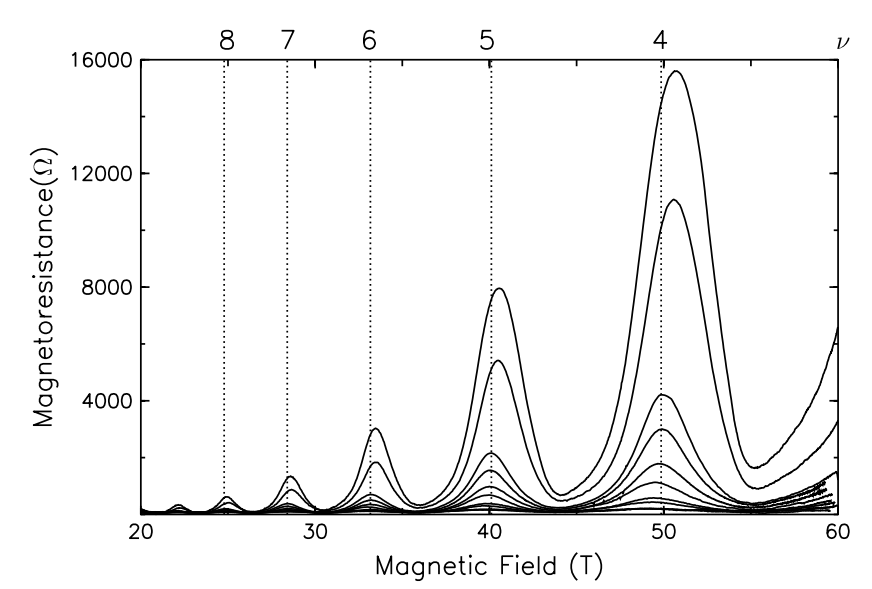

FIG. 1. The temperature dependent magnetoresistance in $\beta^{\prime \prime}$-(BEDT-TTF) ${ }_{2} \mathrm{SF}_{5} \mathrm{CH}_{2} \mathrm{CF}_{2} \mathrm{SO}_{3}$ (from the top, 0.59, 0.94, $1.48,1.58,1.91,2.18,2.68,3.03,3.38,3.80$, and $4.00 \mathrm{~K})$. The dotted lines and numbers indicate integer Landau-level filling factors $\nu=F / B$.

real physical meaning [3,13]. In Ref. [13], it was shown that in such situations, $\rho_{z z}$ behaves in an insulating fashion whenever $\mu$ resides in a Landau gap, which occurs when $\nu=F / B$ is an integer. It is for this reason that the large peaks in $\rho_{z z}$ occur close to integer $\nu$ in Fig. 1; if one incorrectly extracted $\sigma$ from the $\mathrm{SdH}$ data [12], this effect might well lead one to suspect that a "field-induced insulator" [12] had occurred.

The effective mass $m^{*}$ and Dingle temperature $T_{\mathrm{D}}[2,14]$ were deduced from $\mathrm{SdH}$ oscillations between 2 and $6 \mathrm{~T}$, measured in the quasistatic magnet. This corresponds to $59 \leq \nu \leq 33$, where the application of 2D LifshitzKosevich (LK) analysis is able to extract reliable values of $m^{*}[2,3]$. The values deduced are $m^{*}=(1.96 \pm 0.05) m_{\mathrm{e}}$ and $T_{\mathrm{D}}=0.82 \mathrm{~K}$, similar to those found in previous works [7-9,11]. Using $T_{\mathrm{D}}=\hbar / 2 \pi k_{\mathrm{B}} \tau$ [14], the scattering time $\tau$ is $1.22 \times 10^{-12} \mathrm{~s}$, leading to a half-width broadening of the Landau levels $[2,14] \delta E=\hbar \tau^{-1}=0.44 \mathrm{meV}$. By comparison, in the absence of broadening, the energy gap between adjacent Landau levels is $\hbar \omega_{\mathrm{c}}=\hbar e B / \mathrm{m}^{*}$; using $m^{*}$ from the LK analysis, $\hbar \omega_{\mathrm{c}}=2.94 \mathrm{meV}$ at $49.85 \mathrm{~T}$ $(\nu=4)$.

At the maxima close to integer $\nu, \rho_{z z}$ increases exponentially with decreasing $T$. Figure 2 shows $\log \sigma_{z z}=$ $\log \left(1 / \rho_{z z}\right)$ at these points versus $1 / T$. For $1.4<T<$ $3.9 \mathrm{~K}$ the data show clear thermal activation behavior,

$$
\sigma_{z z} \propto e^{-\Delta / k_{\mathrm{B}} T}
$$

at lower $T$, the drop in conductivity saturates. The slope of each line in Fig. 2 gives $\Delta$, and the inset shows $E_{\mathrm{g}}=2 \Delta$ versus $B$ (the reason for plotting $2 \Delta$ will become clear below); $E_{\mathrm{g}}$ increases linearly with $B$, suggesting that the gap is related to the Landau quantization.

The possible explanations for this behavior involve the movement of the Landau levels in $\beta^{\prime \prime}$-(BEDT-TTF $)_{2}-$

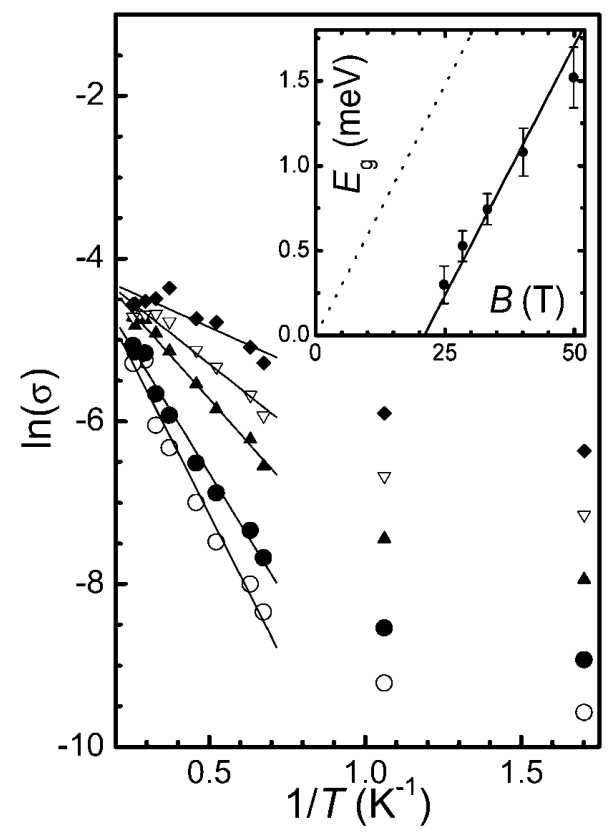

FIG. 2. $\sigma_{z z}$ against $1 / T$ at the magnetoresistance peaks close to integer filling factors; filled diamond $\nu=8$, open triangle $\nu=7$, filled triangle $\nu=6$, filled circle $\nu=5$, and open circle $\nu=4$. The lines are fits used to extract $\Delta$ [see Eq. (1)]. The inset shows the magnetic field dependence of $E_{\mathrm{g}}=2 \Delta$. The dotted line shows $E=\hbar \omega_{\mathrm{c}}=\hbar e B / \mathrm{m}^{*}$ for comparison, and the solid line shows $E=\hbar \omega_{\mathrm{c}}-E_{\mathrm{O}}$, where $E_{\mathrm{O}}$ is a constant offset energy (see text).

$\mathrm{SF}_{5} \mathrm{CH}_{2} \mathrm{CF}_{2} \mathrm{SO}_{3}$ with respect to $\mu$ as a function of $B$. In a perfect $(\tau=\infty)$ metal comprising a single Q2D Fermi surface at $T=0, \mu$ is always pinned to a Landau level in a magnetic field [2]. With increasing $B, \mu$ moves up in a particular Landau level until the degeneracy of the levels below has increased sufficiently for them to accommodate all the quasiparticles. At this point, $\mu$ drops discontinuously into the Landau level below [2]. The presence of Q1D Fermi sheets, which give a continuous dispersion in a magnetic field [4], modifies this behavior $[3,13]$. In this case, shown in Fig. 3, $\mu$ (solid line) is alternately pinned to a Landau level or to the Q1D density of states as the field increases [13]. While $\mu$ is pinned to a Landau level (region $\gamma$ in Fig. 3) the system acts as a Q2D metal. In the region labeled $\delta$ (Fig. 3), $\mu$ is between two adjacent Landau levels, and here only the Q1D dispersion contributes to the conduction. It is in the latter regions that we observe insulating behavior, implying that the Q1D Fermi sheets do not contribute to $\sigma_{z z}$ at low $T$ and high $B$. Q1D quasiparticles are expected to undergo magnetic-field-induced localization [5] at high fields. However, this cannot explain the data. Localization will lead to either a broad band of immobile states or the collapse of the Q1D states onto states of discrete energy [15]. The former would lead to conduction at integer $\nu$ in which quasiparticles were thermally excited from the band of immobile states to the Landau levels; this would 


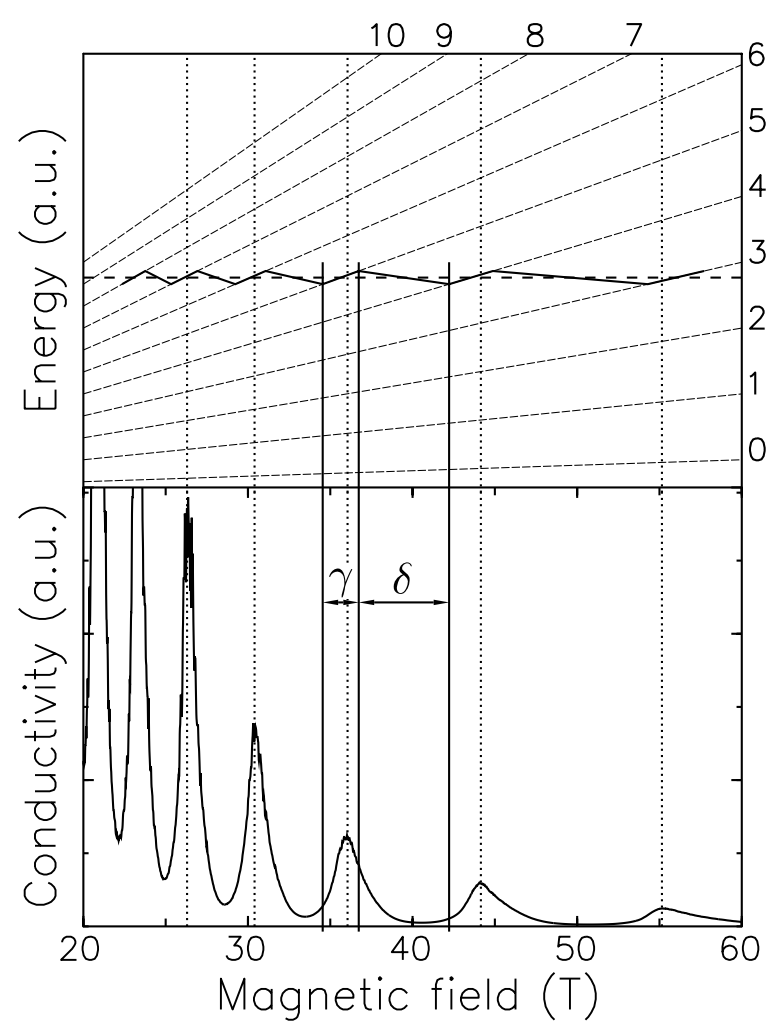

FIG. 3. The upper figure shows the Landau fan diagram with a schematic view of the motion of the chemical potential $\mu$ where $T$ and $\tau^{-1}$ are zero. The solid line indicates the motion of $\mu$ when it is alternately pinned in Q1D and Q2D states. The dashed line shows $\mu$ held constant by, e.g., an incommensurate density wave. The lower part shows the magnetoconductivity oscillation at $500 \mathrm{mK}$; the conductivity peaks whenever $\mu$ is at the center of a Landau level.

result in conductivity obeying a power law. The latter scenario removes the mechanism for pinning $\mu$ between Landau levels, and $\mu$ would instead drop discontinuously between the levels.

A clue to the cause of the activated behavior is given by Fig. 4, which shows the $T$ dependence of $\rho_{z z}$ at $B=0 ; \rho_{z z}$ initially decreases with decreasing $T$ until $T \approx 140 \mathrm{~K}$, where there is a minimum. Thereafter, $\rho_{z z}$ increases with decreasing $T$ until about $35 \mathrm{~K}$, from where it drops to the superconducting transition at $T \approx 5 \mathrm{~K}$. In the region $35<T<140 \mathrm{~K}$, the measured $\rho_{z z}$ depends on the current used in the experiment and can exhibit jumps, bistability (see inset), and hysteresis under certain conditions of bias. The latter behavior and the minimum at $140 \mathrm{~K}$ are typical of organic density-wave (DW) systems, e.g., (BEDT-TTF $)_{3} \mathrm{Cl}_{2} \cdot 2 \mathrm{H}_{2} \mathrm{O}$ [16] [however, in $\beta^{\prime \prime}$-(BEDT-TTF) ${ }_{2} \mathrm{SF}_{5} \mathrm{CH}_{2} \mathrm{CF}_{2} \mathrm{SO}_{3}$ the behavior is somewhat less extreme than that of (BEDT-TTF $)_{3} \mathrm{Cl}_{2} \cdot 2 \mathrm{H}_{2} \mathrm{O}$ [16], presumably because the Q2D pockets of the FS survive the transition]. We therefore propose that the Q1D Fermi sheets in $\beta^{\prime \prime}$-(BEDT-TTF $)_{2} \mathrm{SF}_{5} \mathrm{CH}_{2} \mathrm{CF}_{2} \mathrm{SO}_{3}$ nest to form a DW state at $T \approx 140 \mathrm{~K}$. A DW transition

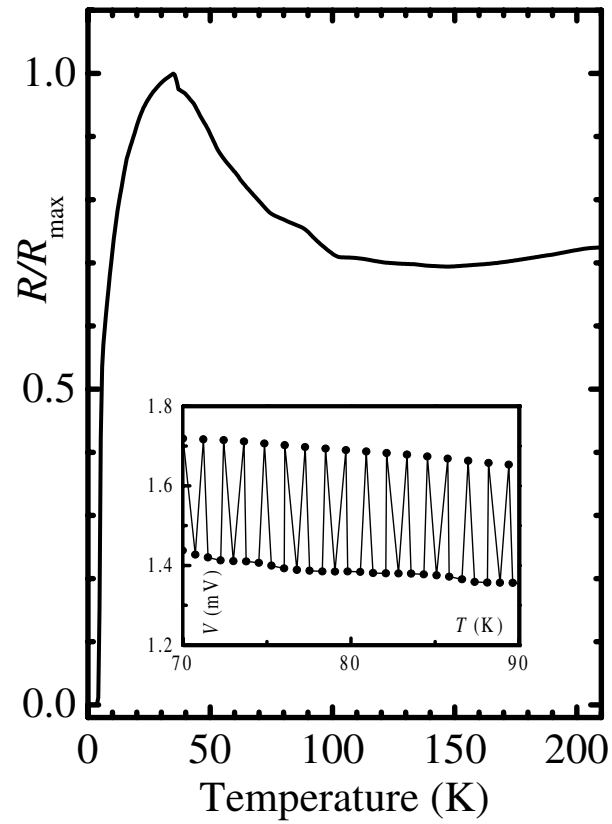

FIG. 4. The temperature dependence of the interlayer resistance $\left(\propto \rho_{z z}\right)$ normalized to its maximum value, measured with a current of $0.5 \mu \mathrm{A}$. With currents any higher than this, $R$ exhibits bistability and hysteresis between 35 and $140 \mathrm{~K}$. The inset shows the sample voltage during a typical region of bistability.

is known to occur at much lower $T$ in the isostructural salt $\beta^{\prime \prime}$-(BEDT-TTF $)_{2} \mathrm{AuBr}_{2}$ [17]; however, the nesting is imperfect, leading to a low-temperature FS comprising a number of small Q2D pockets in addition to an elongated pocket similar to that found in $\beta^{\prime \prime}$-(BEDT-TTF) ${ }_{2} \mathrm{SF}_{5^{-}}$ $\mathrm{CH}_{2} \mathrm{CF}_{2} \mathrm{SO}_{3}$ [17]. In $\beta^{\prime \prime}$-(BEDT-TTF $)_{2} \mathrm{SF}_{5} \mathrm{CH}_{2} \mathrm{CF}_{2} \mathrm{SO}_{3}$, the lack of such additional pockets and the higher ordering $T$ suggest that the nesting is more efficient. The nesting would explain the absence of any signature of the Q1D sheets in the AMRO [8,10] and millimeter-wave data [11].

It is known that incommensurate DWs adjust their nesting vectors $\mathbf{Q}$ in a magnetic field in order to minimize the total free energy [18]. In the Bechgaard salts, this is lowest whenever $\mu$ is situated in a Landau gap of the pocket created by imperfect nesting of the Q1D sheets [18]. This mechanism will not, however, operate if the nesting is perfect [19]. On the other hand, it was recently shown that the oscillations of $\mu$ originating from Landau quantization of additional Q2D FS sections also affect the free energy [19]. Two qualitatively different types of behavior can result [19]; in the case of a commensurate DW, the DW can become unstable to the oscillations of $\mu$. In the incommensurate case, however, $\mathbf{Q}$ is free to adjust itself to prevent the variation of $\mu$ and thereby lower the total free energy. In the case of the DW this is achieved by suppressing the positive $\mu^{2}$ term, while for the $2 \mathrm{D}$ pocket this is achieved by shifting $\mu$ into a Landau gap. The area of reconstructed Brillouin zone determined by $\mathbf{Q}$ will be related in an integral manner to the number of particles contained within 
the 2D pocket, while the particle number associated with the Q1D sheets is strictly zero. This mechanism becomes especially effective if the nesting is perfect so that there is no residual pocket created by the Q1D sheets. In effect, by adjusting $\mathbf{Q}$, a DW is able to compensate for the oscillations in the filling of the Q2D pocket (which result from the fact that $\mu$ is constant), thereby functioning in the same way as an infinite charge reservoir [19]. We believe that this is happening in the present case.

If $\mu$ remains constant, and the Landau levels sweep through it (as shown by the dashed line in Fig. 3), there will be no additional quasiparticle states between the Landau levels; the only means of conduction at integer $\nu$ is therefore thermal excitation of quasiparticles from the full Landau level below $\mu$ (leaving behind holes) into the empty level above it. If the DW gap is much larger than the Landau gap (as suggested by the transition temperature), the Q1D sheets will contribute only to the conductivity at much higher $T$. The situation is analogous to an intrinsic semiconductor; the conductivity is proportional to the number $n$ of quasiparticles excited, which in turn is given by the law of mass action [1], $\sigma_{z z} \propto n \propto e^{-E_{\mathrm{g}} / 2 k_{\mathrm{B}} T}$, where $E_{\mathrm{g}}$ is the energy gap between the filled and empty Landau levels. Comparing this with Eq. (1), we make the identification $2 \Delta \equiv E_{\mathrm{g}}$.

The inset of Fig. 2 shows $E_{\mathrm{g}}$ versus $B$ plotted with the function $E=\hbar \omega_{\mathrm{c}}-E_{\mathrm{O}}$ (solid line); with $E_{\mathrm{O}}$ set to $1.23 \mathrm{meV}$, the experimental values of $E_{\mathrm{g}}$ all lie close to this line, strongly supporting our proposal that $E_{\mathrm{g}}$ is related to the gap between the Landau-level centers, $\hbar \omega_{\mathrm{c}}=\hbar e B /$ $m^{*}$. Landau-level broadening will cause a reduction of the effective energy gap, and it is this reduction that we identify with $E_{\mathrm{O}} ; E_{\mathrm{O}}$ is about 3 times the Landau-level half-width of $0.44 \mathrm{meV}$ deduced from $T_{\mathrm{D}}$. We therefore propose that $\hbar \omega_{\mathrm{c}}-E_{\mathrm{O}}$ represents the threshold energy above which a significant number of thermally excited quasiparticles can contribute to the conductivity.

In a Lorentzian Landau-level density of states $[2,13]$, the tails of the levels extend beyond $\hbar \tau^{-1}$ from the center; even when $\mu$ lies between two Landau levels, there will be a small number of current-carrying quasiparticles. This is probably why the conductivity saturates at low $T$; the carriers in the tails of the Landau levels play the role of extrinsic carriers in a semiconductor system [1].

Finally, we note that the width of the "tail" of the Fermi-Dirac distribution function [2] $\left(\sim 6 k_{\mathrm{B}} T\right)$ is still comparable to the Landau-level width $\hbar / \tau \approx 0.44 \mathrm{meV}$, even at $T \approx 590 \mathrm{mK}\left(6 k_{\mathrm{B}} T \sim 0.3 \mathrm{meV}\right)$. This causes the resistivity at half-integer filling factors to increase with decreasing $T$ [20], but to a much lesser extent than at integer filling factors (Fig. 1). This effect is well documented in the interlayer magnetoresistance of semiconductor super- lattices (see, e.g., [20]) and is not associated with fieldinduced localization [12].

In summary, we have observed thermally activated conductivity at integer Landau-level filling factors in the Q2D organic metal $\beta^{\prime \prime}$-(BEDT-TTF $)_{2} \mathrm{SF}_{5} \mathrm{CH}_{2} \mathrm{CF}_{2} \mathrm{SO}_{3}$. To our knowledge, this is the first identification of such a mechanism in a metallic system. The activation energies deduced from the conductivity are in good agreement with the Landau-level spacings once broadening is taken into account suggesting that the recent identification of a fieldinduced insulator [12] in this material is incorrect. To account for this behavior and for magnetization and resistivity data, we propose that the Q1D sheets of the Fermi surface of $\beta^{\prime \prime}$-(BEDT-TTF) ${ }_{2} \mathrm{SF}_{5} \mathrm{CH}_{2} \mathrm{CF}_{2} \mathrm{SO}_{3}$ are nested. The temperature dependence of the resistivity suggests that this occurs at $T \approx 140 \mathrm{~K}$.

This work is supported by EPSRC (UK). NHMFL is supported by the U.S. Department of Energy (DoE), the National Science Foundation, and the State of Florida. The work at Argonne is sponsored by the DoE, Office of Basic Energy Sciences, Division of Materials Science under Contract No. W-31-109-ENG-38. We thank James Brooks for stimulating discussions.

[1] N. W. Ashcroft and N. D. Mermin, Solid State Physics (Saunders, Philadelphia, 1976).

[2] D. Shoenberg, Magnetic Oscillations in Metals (Cambridge University Press, Cambridge, 1984).

[3] J. Singleton, Rep. Prog. Phys. 63, 1111 (2000).

[4] V. M. Yakovenko and H.-S. Goan, Phys. Rev. B 58, 8002 (1998); A. Ardavan et al., Phys. Rev. B 60, 15500 (1999).

[5] N. Dupuis and G. Montambaux, Phys. Rev. Lett. 68, 357 (1992); Phys. Rev. B 46, 9603 (1992).

[6] U. Geiser et al., J. Am. Chem. Soc. 118, 9996 (1996).

[7] J. Wosnitza et al., Phys. Rev. B 61, 7383 (2000).

[8] D. Beckmann et al., Eur. Phys. J. B 1, 295 (1998).

[9] X. Su et al., Phys. Rev. B 59, 4376 (1999).

[10] M. S. Nam et al., J. Phys. Condens. Matter 13, 2271 (2001).

[11] J. M. Schrama et al., J. Phys. Condens. Matter 13, 2235 (2001).

[12] J. Wosnitza et al., Phys. Rev. Lett. 86, 508 (2001).

[13] N. Harrison et al., Phys. Rev. B 54, 9977 (1996).

[14] M. Springford, Electrons at the Fermi Surface (Cambridge University Press, Cambridge, 1980).

[15] Metal-Insulator Transitions, edited by N. F. Mott (Taylor and Francis, London, 1990).

[16] W. Lubczynski et al., J. Phys. Condens. Matter 8, 6005 (1996).

[17] A. A. House et al., Phys. Rev. B 53, 9127 (1996).

[18] J. R. Cooper et al., Phys. Rev. Lett. 63, 1984 (1989); S. T. Hannahs et al., Phys. Rev. Lett. 63, 1988 (1989).

[19] N. Harrison, Phys. Rev. Lett. 83, 1395 (1999).

[20] D. P. Druist et al., Phys. Rev. Lett. 80, 365 (1998). 